\title{
Racionalidad de las Decisiones Judiciales y For- mulación de Argumentos en Litigio
}

\author{
Rationality of Judicial Decisions and Formulation of \\ Arguments in the Litigation
}

\section{Renato Mármol Álvarez}

Investigador Jurídico, Vaca \& Asociados

Artículo Original (Revisión)

RFJ, No. 1, 2017, pp. 81-94, ISSN 2588-0837

RESUMEN: El documento tiene como objetivo establecer parámetros básicos en la aplicación de la lógica en la formulación de argumentos jurídicos. A su vez, persigue describir los tipos de sofismas y falacias presentes en el razonamiento jurídico a partir de la incorporación de aportes del Análisis Económico del Derecho y la escuela de Crítica Legal.

PALABRAS CLAVE: Racionalidad, decisiones judiciales, argumentos, razonamiento jurídico, litigio.

ABSTRACT: The document aims to establish basic parameters in the application of logic in the formulation of legal arguments. In addition, it seeks to describe the types of fallacies present in legal reasoning based on the incorporation of contributions from the Economic Analysis of Law and the School of Legal Criticism.

KEY WORDS: Rationality, judicial decisions, arguments, legal reasoning, litigation

\section{INTRODUCCIÓN}

La Lógica Jurídica en la actualidad es considerada como un campo de dominio obligatorio para todo jurista o individuo que aspire a ejercer la profesión. La capacidad de razonar y entender el Derecho y sus dinámicas es la principal distinción existente entre abogados y profesionales de otras ramas. 
La idea del jurista conocedor de absolutamente todas las leyes referentes a un determinado tema está cada día más lejos de la realidad, especialmente hoy que los ordenamientos jurídicos se caracterizan por intentar regular todos los aspectos posibles de la conducta humana. El abogado progresivamente ha dejado el rol de ser un mero agente jurídico y se ha orientado a ser un analista e intérprete de las disposiciones del poder legislativo. Esto, sin embargo, genera incertidumbre en la población y hace meritoria la pregunta: ¿ En la actualidad el razonamiento aplicado por los juristas es el idóneo para solucionar los conflictos de la sociedad en la que ejerce sus funciones?

Los principios y fundamentos de la lógica aristotélica que se enseña con fervor en las facultades de jurisprudencia son en la actualidad la base del modo de pensar y actuar de la mayoría de los abogados y jueces. La normalización de estos principios, lamentablemente, trae consigo desventajas derivadas precisamente de la antigüedad de este sistema. Sin embargo, varios juristas aún la defienden; posiblemente movidos por sus propias costumbres y un sentimiento de nostalgia que les hace justificar la aplicación de un sistema arcaico con el argumento de que es una alternativa pragmática y adaptable. Sobre ello García Murillo y García Ramírez afirman: "La lógica y su aplicación a la ciencia jurídica da por resultado una liberación de rigidez que la hace adecuada para una nueva sociedad que se transforma insensatamente" (García Murillo \& García Ramírez, 2011).

A pesar de que en la sociedad actual se evidencien con fuerza las falencias de la lógica jurídica clásica, es meritorio resaltar que el debate sobre la eficiencia y la legitimidad científica del razonamiento deductivo no es algo nuevo en el Derecho.

Los cambios estructurales en la sociedad, afianzados por los avances tecnológicos y los conflictos bélicos, se extendieron a las ciencias jurídicas y motivaron una serie de nuevas corrientes de pensamiento cuyo fin es actualizar la forma de hacer Derecho. Entre las principales corrientes jurídicas que surgieron como respuesta a la técnica jurídica clásica destacan el Análisis Económico del Derecho (AED) y los Estudios Críticos Legales (CLS), que surgieron en distintas etapas del siglo pasado con propuestas revolucionarias del concepto clásico de Derecho. La primera ofreció una visión pragmática y mercantilista de la práctica jurídica, buscando justificarla en preceptos econométricos, mientras que la segunda recriminó los abusos de poder de los juristas, a quienes instó a dejar atrás la visión elitista de los abogados. 
Es en medio de esta discusión que surge el presente artículo, que de forma general tiene como objetivo establecer parámetros básicos en la aplicación de la lógica en la formulación de argumentos jurídicos, especialmente por parte de los jueces al dictar sentencia. A su vez, busca desmenuzar los principales tipos de sofismas y falacias presentes en el razonamiento jurídico, discutir parámetros de optimización de la argumentación judicial, y diferenciar de forma detallada los puntos claves de corrientes nuevas como el Análisis Económico del Derecho y los Estudios Críticos Legales con relación a la técnica jurídica clásica.

\section{FALACIAS Y SOFISMAS EN EL RAZONAMIENTO JURÍDICO}

Cuando se busca discutir sobre la validez de un argumento, es prácticamente imposible evitar que las falacias aparezcan en medio del debate. Desmenuzar los principales tipos de sofismas y falacias presentes en el razonamiento jurídico se convierte en una necesidad antes de poder debatir sobre la argumentación. Aunque estos términos son de difícil definición, parece existir un consenso al hablarse de falacias, haciéndose mención a argumentos que "violan las reglas lógico-formales, materiales o dialécticas de la argumentación clásica de forma sistematizada" (Cárdenas García, 2015).

En el Derecho, la aplicación de falacias y sofismas es recurrente y reiterada, especialmente en el litigio administrativo y penal donde las partes argumentan de forma que salgan favorecidas incluso cuando omiten estructuras lógicas en sus pretensiones. Bajo esa premisa, es posible reconocer y definir algunas de las falacias más populares y reconocidas en el campo jurídico. Entre ellas, la más común al momento de litigar es la falacia Ad Hominem, cuyo nombre fue acuñado por vez primera en el siglo XVII por el célebre filósofo John Locke, y que es descrita actualmente como: "aquella que se produce cuando el peso de un argumento no intenta refutar al argumento mismo, sino a la credibilidad del oponente, bien, tratando de eliminar sus puntos de vista, o presentándolo como alguien poco inteligente, confiable, inconsecuente, sesgado, etc." (Gutiérrez Cham, 2007: 70)

Esta falacia, de forma simplificada, es como se denomina a un argumento que no responde al problema planteado y, en su lugar, busca desprestigiar y desmeritar a la contraparte en el debate. En el Derecho es bastante común y forma parte incluso de la estrategia de varios abogados litigantes, tanto en el área civil como penal. También se manifiesta 
en los debates en el área legislativa, por motivaciones políticas. El litigio y el debate, como ejercicios realizados en tiempo real con poco tiempo de reacción, permite que este tipo de argumentos pasen como válidos y den una ventaja potencial e injusta a un argumento sobre otro.

La siguiente falacia más común en el campo judicial es la denominada Petición de Principio, muy recurrente en las decisiones judiciales: "Un argumento comete una petición de principio cuando su conclusión está incluida en sus premisas" (Vilaró, 2010). De la misma forma que la Falacia ad Hominem, la Petición de Principio puede pasar desapercibida por las partes en medio del litigio oral. Un juez con poco conocimiento de doctrina o de lógica es capaz de admitir y utilizar una premisa para justificar la misma idea planteada de forma distinta.

Este tipo de razonamiento genera graves contradicciones, y es capaz de hacer desviar al juez de hacer cumplir la finalidad última del Derecho, que es la Justicia.

Las falacias Ad Hominem y la Petición de Principio, aunque son las que más se pueden apreciar como parte de la técnica jurídica actual, no son las únicas. Un argumento demasiado ambiguo, cosa extremadamente normal al momento de formular pretensiones en el campo del Derecho, es a su vez un argumento falaz. De la misma forma, según Reñon, 2010, argumentos político y jurídico caen en otras falacias comunes y apelan al poder de quien formuló la idea (Ad Verecundiam), a los sentimientos y la piedad del juez (Ad Misericordiam), a la normalidad de un acto entre la gente de un colectivo más allá de que sea correcto o no (Ad Populum) o incluso a la fuerza sin admitir ningún tipo de negociación (Ad Baculum).

Lamentablemente, los juristas que aplican con cotidianidad este tipo de argumentos abundan en todas las áreas del Derecho y son ellos precisamente los que dan mala reputación a las ciencias jurídicas en la sociedad. La aplicación de este tipo de prácticas engañosas pervierte la finalidad del Derecho y convierte el ejercicio jurídico en una herramienta de poder que favorece a aquellos que no tienen reparos morales para evitar la utilización de este tipo de argumentos para ganar un caso.

Sin embargo, la argumentación mantiene otros varios problemas. Los sofismas, por ejemplo, destruyen los sistemas lógicos clásicos y pervierten el ejercicio de debate. Estos argumentos, generalmente falsos, se basan en la dialéctica y toman como objetivo justificar ideas 
falaces. Los sofismas buscan inducir al oponente al error mediante provocaciones y el hacer que en el debate se tome como real un argumento que no está comprobado. Estos se remontan hasta la época de Platón y Aristóteles, y fueron aplicados por pseudo-filósofos para convencer a la gente de su superioridad.

La aplicación de los sofismas en el litigio es capaz de dañar a una de las partes al punto de hacerle perder un caso, y si es usada por un juez para la resolución de un conflicto podría ir esa sentencia o auto resolutorio en contra de la solución lógica del caso. Las consecuencias empeoran si llegara a pensarse en el juez como un actor más, con una ideología determinada e intereses y no como un árbitro imparcial como idealmente debería ser. Bajo condiciones ideales, el juez actuaría de forma neutral y resolvería únicamente teniendo como base los distintos argumentos planteados durante el proceso. Esta probidad, lamentablemente, es cada vez menos normal: los jueces en ocasiones actúan bajo su propio beneficio, y el uso de falacias permite justificar de una forma u otra las decisiones que toman. En vez de resolver el caso sobre la base de una motivación sustentada en pruebas y elementos de hecho y de derecho, el juez construye la motivación sobre la base de una resolución que ya tomó incluso antes de escuchar a las partes. Aún cuando estas posibles soluciones podrían ser aparentemente injustificables, las falacias y los sofismas son capaces de realizarlo y pasar inadvertidas para las partes si estas no tienen a su vez conocimiento de su importancia. Es así como se burla la seguridad jurídica de las partes y se destruye la idea del Derecho como un sistema íntegro que busca el bien común de los miembros de la sociedad.

Es bajo estos parámetros que se pueden definir las falacias y los sofismas. Resulta más que obvio el hecho de que el razonamiento jurídico debe librarse de estos elementos. Sin embargo, es también necesario brindar alternativas al juez para fundamentar sus decisiones. La libertad de formular soluciones a problemas de carácter jurídico únicamente con lo que dice la norma y su propia interpretación es excesiva y causante varios de los problemas que tiene el Derecho hoy en día. Es precisamente motivado en esto que se desarrollan nuevas corrientes jurídicas que buscan modernizar las técnicas de hacer Derecho. 


\section{MODERNIZACIÓN DEL RAZONAMIENTO JURÍDICO Y LA BÚSQUEDA DE JUSTICIA}

Discutir parámetros de optimización de la argumentación judicial no solo se ha vuelto una parte de la agenda jurídica, sino una prioridad en todo sentido. Los elementos expuestos anteriormente como deficiencias del razonamiento no son nuevos para el Derecho.

Los sofismas y las falacias han sido aplicadas en las decisiones judiciales por siglos como herramientas de los jueces para fallar de forma arbitraria. Como consecuencia, varias olas de nuevas corrientes y pensadores critican la forma de hacer Derecho en todo el mundo, y el ejemplo máximo puede ser la Corriente de los Estudios Críticos Legales al establecer que "el sistema que se enseña a los estudiantes de Derecho es aberrante, absurdo y está politizado por el entorno sociocultural y económico que los rodea" (Kennedy, 2004:34). Esta crítica demuestra el descontento general de los mismos juristas con la forma en la que se maneja el entorno de los profesionales del Derecho en la actualidad. Las ciencias jurídicas son acusadas de ya no responder al fin de la justicia y el bien común.

Es a partir de este descontento que se extienden varias de las críticas que se contraponen a la técnica jurídica que se aplica en la actualidad. Entre las más relevantes se puede identificar la ya mencionada deficiencia de fundamentación científica en la toma de decisiones judiciales.

El uso de falacias genera rechazo en la comunidad jurídica y es considerada un verdadero retroceso en las sociedades donde se aplica el Derecho anglosajón. En este contexto, uno de los mayores problemas encontrados es que "Los razonamientos jurídicos suelen ser una suerte de razonamientos sobrenaturales y circulares, porque usan conceptos creados para crear otros conceptos" (González \& Lean, 2009). Al ser una ciencia que creó su propio lenguaje y que trabaja de forma independiente al esto de las Ciencias Sociales, el Derecho es propenso a que sus argumentos se vuelvan circulares y caigan especialmente en la ya mencionada falacia de Petición de Principio. Esta falla surge de las normas definitorias que tanto abundan en nuestro ordenamiento jurídico. Al desarrollarse un lenguaje jurídico basado en términos definidos por el mismo lenguaje jurídico, todo razonamiento que se realice en este lenguaje se justificará en un sistema eminentemente circular. Esto limitaría a la lógica jurídica dentro del sistema autoreferente del Derecho y eliminaría toda posibilidad de 
ver al razonamiento jurídico en general más allá de una disciplina intrínseca de la educación legal.

Esto es explicado de mejor manera cuando se plantea como el mayor problema de la actual forma de hacer Derecho en el mundo su incapacidad de relacionarse con otras ramas del conocimiento. Es así como se describe:

"La mayor dificultad cuando se busca generar trabajos interdisciplinarios entre las ciencias sociales y jurídicas es precisamente sobreponer los problemas epistemológicos surgidos del metalenguaje del Derecho, la inconmensurabilidad de paradigmas entre los distintos tipos de ciencias y los sistemas cerrados autopoiéticos de la jurisprudencia." (Salas, 2006)

El enfoque clásico y defensor de los principios Aristotélicos en la Lógica Jurídica también es partidaria de la idea del Derecho como un sistema cerrado. La visión clásica positivista niega la posibilidad de que las Ciencias Jurídicas trabajen con otras ciencias hermanas como son las Ciencias Políticas, la Sociología, la Antropología, la Historia o incluso la Economía. Esta postura arcaica es la que ha puesto en duda el mismo término de "Ciencias Jurídicas"; al momento de cerrar a las ciencias jurídicas de su relación con otras ciencias, se elimina simultáneamente toda posibilidad de que la Lógica Jurídica pueda ser considerada una ciencia de forma autónoma; y contradice la visión clásica que considera a la Lógica Jurídica científicamente fundamentada. Si hay manera de calificar a la lógica jurídica, sería como la lógica de lo justo. Es una lógica volcada a su finalidad de encontrar una conclusión o solución justa a un problema planteado. Lamentablemente, estos pensadores parecen no tomar en cuenta que si la Lógica Jurídica es aquella que rige los presupuestos de lo que es justo y lo que no, es necesario permitir que la misma evolucione y se compatibilice con la sociedad actual.

Por su parte, el trabajo conjunto de las Ciencias Jurídicas con otras ciencias y disciplinas es uno de los objetivos que tienen los nuevos doctrinarios y filósofos en sus nuevas corrientes. Más aún cuando el Derecho no responde únicamente a la idea de los mecanismos de resolución judicial.

La lógica jurídica y el Derecho necesitan una reconstrucción transversal y un análisis desde sus mismos ejes, que debe partir de la misma idea de la Justicia como fin deontológico de las Ciencias Jurídicas 
en general. Precisamente, estos razonamientos circulares y sobrenaturales dan paso a un abuso de la ley y a la posibilidad de que el poder judicial pueda fallar de forma arbitraria y beneficiar a una de las partes según la conveniencia de quien juzga. El juez se convierte en cierta forma en una suerte de ser omnipotente capaz de administrar justicia e interpretar la ley de la forma que él crea conveniente sin necesidad de responder ante nadie.

Así, por ejemplo, antes de describir al Análisis Económico del Derecho o a los Estudios Críticos Legales, es necesario profundizar la motivación de encontrar nuevas formas de hacer Derecho. Varios de los partidarios y catedráticos en extremo afines a la técnica convencional insisten en que no debería cambiarse, e incluso buscan salidas intermedias como una reforma o una reestructuración. "La lógica jurídica tiene un papel primordial en las ciencias jurídicas y sociales, y es necesario difundir sus contenidos a los jueces, los abogados litigantes, profesionales del derecho en general y estudiantes, para que los apliquen" (Rosales Gramajo, 2010).

Las posturas de los adeptos a esta forma de hacer Derecho también responden a la interrogante que surge sobre su validez científica, así como al debate sobre su relevancia entre jueces y juristas en general. Según esta visión, la Lógica Jurídica se puede describir como una ciencia dedicada al estudio de la metodología que estructura la unión de elementos en la formulación de un argumento jurídico. Este intento de dar una fundamentación científica busca legitimarla como el mecanismo más idóneo para el análisis del Derecho.

A pesar de este tipo de admiradores y seguidores, la Lógica Jurídica pierde veracidad cada día y en algún punto quedará obsoleta de no adaptarse a la realidad.

En primera instancia, se debe buscar fundamentar el raciocinio y la argumentación jurídica en principios verdaderamente científicos; aspectos que deben mencionarse antes de dialogar sobre los mecanismos de modernización de la lógica jurídica y el Derecho en forma general, tales como su fin último: la justicia. Los conceptos de "justicia" han cambiado con los años, y sus cambios son a su vez generadores de cambios en el mismo Derecho. Resulta meritorio revisar de forma breve las definiciones de "justicia" con las que se ha trabajado a lo largo de la historia para que haya un mejor entendimiento de las nuevas formas de hacer Derecho que se buscará describir. 
Inicialmente, la Justicia partió de una visión moralista que planteó preceptos religiosos en normas ambiguas que oscilaban entre un carácter jurídico y uno divino. El iusnaturalismo entero partió de que la idea de la justicia nacía en la diferencia entre lo que es bueno y lo que es malo; tuvo sus leves modificaciones y cambios a través de la historia y desembocó en las teorías tanto aplicadas en la Roma antigua como en la Edad Media con la Escuela Escolástica. Esta se define como un sistema que "desciende desde los principios más abstractos a las conclusiones que se desprenden de ellos y que tienen una validez condicionada por la materia social mutable en la cual pretenden regir" (Pérez, 1966:594); lo cual cambia en la Edad Moderna con la Revolución Industrial y el nacimiento de las ideas de izquierda. Mientras muchos pensadores socialistas hablan sobre conceptos como la justicia social o la justicia de clases, es preciso señalar que el presupuesto de Marx versaba sobre que "una sociedad comunista plenamente desarrollada parece situarse "más allá de la justicia”". (Guiñazú, 2001:150)

Así, Marx puede que jamás haya trabajado en demasía sobre el concepto de Justicia, pero se sabe que él consideraba al Derecho como una herramienta de dominación a favor de las clases dominantes para subyugar y oprimir a las clases débiles, como es el proletariado; la sociedad comunista en contraste a la capitalista sería una sociedad armoniosa donde no haría falta de regulaciones legales al haber justicia y recursos para todos.

Las nuevas corrientes Marxistas denominadas de forma general como corrientes de Marxismo analítico o Marxismo crítico buscan definir estos conceptos y trabajar más en nuestra realidad de lo que hizo el autor de "El Capital".

Por lo expuesto, se hace meritorio un análisis introspectivo de las ciencias jurídicas en general y de la lógica jurídica de forma específica. El marxismo crítico planteado por Kennedy o el Análisis Económico del Derecho surgen en el siglo XX y continúan su evolución en la primera década del siglo XXI como mecanismos para definir parámetros mínimos a seguir por parte de todo funcionario al tomar una decisión de carácter judicial. Estos parten de conceptos de Justicia modernos y de Derecho pragmáticos, por lo que buscan afianzar el bien común de la sociedad como su interés máximo. La influencia de autores como John Rawls o Amartya Sen es clara y permite entender de mejor manera la forma de trabajar de los distintos integrantes de la sociedad. 


\section{CORRIENTES NUEVAS DE PENSAMIENTO COMO CON- TRAPOSICIÓN A LA LÓGICA CLÁSICA}

Diferenciar de forma puntual los puntos clave del Análisis Económico del Derecho con la técnica jurídica clásica no es tarea fácil. El Análisis Económico del Derecho (AED) es una corriente que plantea que los abogados estudien las leyes de la misma forma que los economistas estudian al mercado. "El análisis económico del derecho dota y fundamenta a la estructura jurídica para que la eficacia y la eficiencia sean propias del marco que reviste la creación de las leyes" (Gutiérrez O., 2012). La eficiencia de una ley puede ser analizada mediante una revisión de los casos donde ha sido aplicada y las veces que ha sido aplicada. Todo esto hace más fácil el trabajo del juez al permitir que se conjuguen preceptos estadísticos con su propia motivación al dictar sentencia. Incluso en caso de que decida antes de motivar, encontrará sustento en las ciencias exactas para fundamentar su decisión y no en falacias como la Petición de Principio o la falacia Ad Hominem. Esto permite que haya una mayor seguridad jurídica entre las partes y que las resoluciones tengan una mayor validez científica.

Los sistemas expuestos son revolucionarios puesto que erradicarían falacias mientras permiten lo que plantea Di Chiazza, ado por Lionetti, cuando establece que sería la meta del Derecho "buscar -por medio de la interpretación jurídica- el máximo beneficio posible en el marco de las circunstancias del caso, para el mayor número posible de sujetos comprometidos en dicho caso, a los menores costos posibles" (Lionetti de Zorzi, 2006).

El plantear de esta manera el principio de la eficiencia podría ser revolucionario de ser implementado correctamente en las ciencias jurídicas. Esto partiría de una modificación de la forma clásica de verlo: "Eficiencia es entendida como la capacidad de la regla formal para permitir la transferencia de derechos (bienes y servicios) a sus usos más valiosos." (C. North, 1990)

Esto a su vez sería un gran avance al permitir una mejora a la sociedad, al hacer más eficiente funciones del Estado como la Legislativa y la Judicial. Esto permitiría cumplir uno de los retos que tienen los países en vías de desarrollo, que consiste en implementar "procesos de reforma y modernización al Estado, con énfasis en la dimensión económica y administrativa” (Toro Quintana, 2010) 
No es misterio, ya que es necesario reformar la visión de la Lógica Jurídica y revestirla con aplicaciones prácticas. "Incluso cuando sea necesaria la formulación de silogismos y trabajos lógicos, no puede ni debe ser la herramienta principal de los jueces y operadores jurídicos, especialmente cuando hay alternativas nuevas capaces de evitar el uso de sofismas y falacias en el razonamiento jurídico" (Mac Lean, 2013). El Análisis Económico del Derecho se presenta ante el foro de juristas como una respuesta a los problemas de la lógica clásica, puesto que el juez deja de actuar en base a su propio razonamiento y empieza a basarse en presupuestos y cifras estadísticas.

El Derecho se caracteriza por estar un paso por detrás de la realidad. Lamentablemente, los cambios que sufrió el mundo en el último siglo fueron tan radicales que las formas de hacer derecho quedaron obsoletas. Los avances se dan de forma tan veloz que es necesario utilizar alternativas nuevas y suplirse de otras ciencias para alcanzar validez científica.

\section{CONCLUSIONES}

Como palabras finales, es meritorio resaltar la importancia de este debate. Las técnicas jurídicas actuales permiten el uso de falacias y los sofismas en el Derecho. Las falacias están tan institucionalizadas en el trabajo de los juristas que muchos parecen aceptarlos y defenderlos como parte de las ciencias jurídicas. Estas generan daños irreparables en los sistemas jurídicos actuales y son causa de críticas, mala reputación y tergiversación de la finalidad de los abogados en la sociedad. Indudablemente, la sociedad actual no puede ser regulada y enfocada con el mismo lente que juzgó a las personas antes de la Revolución Informática y el inicio de la Era del Conocimiento. Las nuevas corrientes de pensamiento jurídico son, así, una alternativa viable para dar un motivo científico a la forma de actuar y pensar de los jueces en la actualidad.

Es necesario reconocer nuevas alternativas como motor de las decisiones de los jueces. La lógica jurídica requiere una reforma desde sus cimientos y resulta inaceptable que se apliquen principios que permitan la tergiversación de argumentos por parte de los abogados. Las falacias parecen ser parte normal de los procesos de litigio y resultan a su vez en extremo comunes cuando los jueces deciden resolver sobre un tema donde tienen intereses. Visiones como la propuesta por el Análisis Económico del Derecho y la visión estructural del principal 
y el agente permiten un análisis de los casos basado en presupuestos y estadísticas, que mejoran significativamente la forma de resolver un caso y garantizan que tengan un resorte inminentemente científico.

En medio de este caos, el Análisis Económico del Derecho o los Estudios Críticos Legales son alternativas viables para la modernización del Derecho. Las Ciencias Jurídicas no pueden dejar de evolucionar y se requiere un trabajo constante para evitar quedar obsoletos frente a este mundo tan cambiante.

A su vez, es necesario analizar los problemas epistemológicos de la inconmensurabilidad de paradigmas que impiden el trabajo conjunto del Derecho con otras Ciencias y ramas del conocimiento. Las ciencias sociales como la Filosofía, la Sociología, la Historia o la Antropología pueden jugar un papel esencial en el campo de las fuentes del Derecho, el análisis de la doctrina clásica e incluso en la motivación de las decisiones judiciales. Similarmente, la Economía puede ayudar al Derecho a convertirse en un eje viable en nuestra sociedad, pues permitiría que se adapte a una nueva forma de gobernar donde las ciencias políticas, jurídicas y económicas parecen cada vez estar más entrelazadas.

\section{REFERENCIAS BIBLIOGRÁFICAS}

C. North, D. (1990). Institutions, Institutional Change and Economic Performance (1ra ed.). St Louis: Washington University.

Cárdenas García, J. (2015). Los argumentos jurídicos y las falacias. En Metodologías: enseñanza e investigación jurídicas (1ra ed. 153-201). México: UNAM, Instituto de Investigaciones Jurídicas.

García Murillo, J. G. y García Ramírez, V. (2011). Lógica Jurídica. De Jure. Universidad de Colima, No 6. 131-162.

González, A. B. y Lean, A. C. M. (2009). La enseñanza del derecho: ¿cofradía o archicofradía? Academia: Revista sobre enseñanza del derecho de Buenos Aires, Vol. 7, No 13. 21-47. 
Guiñazú, M. C. (2001). Marxismo analítico y justicia: ¿más allá de Rawls? En Teoría y filosofía política: la tradición clásica y las nuevas fronteras (1ra ed. 150-158). Buenos Aires: CLACSO, Consejo Latinoamericano de Ciencias Sociales.

Gutiérrez Cham, G. (2007). Falacia ad hominem en dos periódicos religiosos del siglo XIX en Guadalajara. Una perspectiva pragma-dialéctica. Escritos. Revista del Centro de Ciencias del Lenguaje. 35-36. 61-83.

Gutiérrez O., J. A. (2012). Análisis económico del derecho y del derecho económico bajo revisión empresarial. Opinión Jurídica, Vol. 11, No 21. 117-134.

Kennedy, D. (2004). La educación legal como preparación para la jerarquía. Academia: Revista sobre enseñanza del derecho de Buenos Aires, Vol. 2, No 3. 117-147.

Lionetti de Zorzi, J. P. (2006). Razonamiento jurídico y toma de decisión. Breves notas acerca de la influencia de la racionalidad y la irracionalidad en la decisión judicial. Universitas. Revista de Filosofía, Derecho y Política, No 3. 3-22.

Mac Lean, A. C. (2013). Destrezas legales para la realidad del derecho en el Perú. Sinergia e Innovación, Vol. 1, No 1. 118-135.

Pérez, L. A. (1966). La filosofía jurídica en la patrística y en la escolástica. Revista de la Facultad de Derecho de México, Vol. 1, No 63. 591-612.

Reñón, L. V. (2010). Archivo histórico de textos, I: Falacias. Revista iberoamericana de argumentación, No 1. 142-182.

Rosales Gramajo, F. J. (2010). Lógica Jurídica: Instrumento indispensable para el juez y el abogado litigante (1ra ed.). Guatemala: Instituto de investigaciones jurídicas de la Universidad Rafael Landívar.

Salas, M. E. (2006). Interdisciplinariedad de las ciencias sociales y jurídicas: ¿impostura intelectual o aspiración científica? Revista de ciencias sociales, No 113. 55-69. 
Toro Quintana, G. (2010). La modernización del Estado. En Teoría Política y Gestión Pública (1ra ed. 15-34). Santiago de Chile: KAS, Konrad Aduaneur Stiftung.

Vilaró, I. (2010). Una definición pragmática de la falacia de petición de principio. Areté, Vol. 2, No 1. 107-127.

Recibido: 8/01/2017

Aprobado para publicación: 27/05/2017

Renato Mármol Álvarez: Investigador jurídico Vaca \& Asociados.

Correo electrónico: rmarmol876@puce.edu.ec 